\title{
Performance of Asynchronous MC-CDMA Systems with Maximal Ratio Combining in Frequency-Selective Fading Channels
}

\author{
Keli Zhang \\ School of Electrical and Electronic Engineering, Nanyang Technological University, Nanyang Avenue, Singapore 639798 \\ Email: eklzhang@pmail.ntu.edu.sg \\ Yong Liang Guan \\ School of Electrical and Electronic Engineering, Nanyang Technological University, Nanyang Avenue, Singapore 639798 \\ Email: eylguan@ntu.edu.sg
}

Received 28 February 2003; Revised 29 August 2003

\begin{abstract}
The bit error rate (BER) performance of the asynchronous uplink channel of multicarrier code division multiple access (MCCDMA) systems with maximal ratio combining (MRC) is analyzed. The study takes into account the effects of channel path correlations in generalized frequency-selective fading channels. Closed-form BER expressions are developed for correlated Nakagami fading channels with arbitrary fading parameters. For channels with correlated Rician fading paths, the BER formula developed is in one-dimensional integration form with finite integration limits, which is also easy to evaluate. The accuracy of the derived BER formulas are verified by computer simulations. The derived BER formulas are also useful in terms of computing other system performance measures such as error floor and user capacity.
\end{abstract}

Keywords and phrases: MC-CDMA, MRC, asynchronous transmission, correlations, fading channels.

\section{INTRODUCTION}

Multicarrier code division multiple access (MC-CDMA) is a technique that combines direct sequence (DS) CDMA with orthogonal frequency division multiplexing (OFDM) modulation. It is one of the candidate technologies considered for the 4th-generation wireless communication systems [1]. MC-CDMA transmits every data symbol on multiple narrowband subcarriers and utilizes cyclic prefix to absorb and remove intersymbol interference (ISI) arising from frequency-selective fading. As it is unlikely for all subcarriers to experience deep fade simultaneously, frequency diversity is achieved when the subcarriers are appropriately combined at the receiver. In $[2,3]$, it is shown that MC-CDMA outperforms the conventional DS-CDMA and two other forms of CDMA with OFDM modulation, namely MC-DS-CDMA and multitone CDMA.

Several combining techniques have been proposed for MC-CDMA systems. Maximal ratio combining (MRC) offers maximum improvement in the presence of spectrally white Gaussian noise $[4,5]$. It is shown to achieve better performance for MC-CDMA uplink than equal gain combining (EGC) in [3], and the resultant system has lower error floor than DS-CDMA and MC-DS-CDMA.
The bit error rate (BER) performance of MC-CDMA systems is not easy to analyze as the receiver operations involve coherent combining of a large number of independent or correlated fading subcarriers with possibly different fading statistics. Signal analysis is further complicated by the presence of multiuser interference (MUI) in the received signal. In the literature, simulations are often used to study the performance of MC-CDMA systems $[2,6,7,8]$. For the downlink performance of MC-CDMA with MRC, performance lower bounds are given in [2, 9]. For the MC-CDMA uplink with MRC, to the authors' knowledge, the most general performance analysis is given in [3], where Monte Carlo integration is used to evaluate the BER expressions which involve multidimensional integration of dimensions equal to the number of subcarriers. Although simplified performance formulations are given in $[10,11,12]$, they are based on the assumptions of independent and identically distributed (i.i.d.) fading among the channel paths [10], or independent fading among the subcarriers $[11,12]$. Furthermore, all the works reported in $[2,3,9,10,11,12]$ only consider Rayleigh fading channels.

In this paper, we conduct a BER analysis for MC-CDMA uplink with MRC in Rayleigh, Rician, and Nakagami fading 
channels with arbitrary fading parameters and correlations between the channel paths or subcarriers. A simplified signal model for the MRC output in uplink channel is first developed. By employing the time-frequency equivalence for multicarrier signals, the generic BER expression of the $\mathrm{MC}$ CDMA system with MRC is expressed in terms of the channel impulse response (CIR) information, instead of the subcarrier fading information. Then, by using the technique of Cholesky decomposition, a closed-form BER formula that does not require integration is obtained for channels with correlated Nakagami fading paths. For channels with correlated Rician fading paths, the BER formula is reduced to a form of one-dimensional integration by employing an alternative form of the Gaussian Q-function.

The organization of the paper is as follows. The generic BER analysis is given in Section 2, while specific BER formulations for a variety of fading channels are given in Section 3. Section 4 presents the verification results and Section 5 concludes the paper.

\section{GENERIC BER ANALYSIS}

\subsection{Signal model}

Considering an MC-CDMA system with $N_{u}$ users, each of whom employs $N_{c}$ subcarriers modulated with BPSK, the transmitted signal corresponding to the $k$ th user can be expressed as follows:

$$
\begin{aligned}
& s_{k}(t) \\
& =\sum_{v=-\infty}^{\infty} \sqrt{\frac{2 E_{b}}{N_{c} T_{s}}} \sum_{n=1}^{N_{c}} b_{k}(v) c_{k, n} u_{T_{s}}\left(t-v T_{s}\right) \cos \left(w_{n} t+\theta_{k, n}\right),
\end{aligned}
$$

where $E_{b}$ and $T_{s}$ are the bit energy and symbol duration respectively; $u_{T_{s}}(t)$ represents a rectangular pulse waveform with amplitude 1 and duration $T_{s} ; b_{k}(v)$ is the $v$ th transmitted data bit, $c_{k, n}$ is the random spreading code; $w_{n}$ is the frequency of the $n$th subcarrier; and $\theta_{k, n}$ is the random phase at transmitter.

For uplink transmission, the base station receives signals from different users through different propagation channels. This leads to different channel amplitudes and phases to be associated with different users. More importantly, asynchronous transmission between users results in misalignment in the signal arriving times among different users. Hence the received MC-CDMA uplink signal from a quasistatic frequency-selective fading channel is of the form

$$
\begin{gathered}
r(t)=\eta(t) \\
+\sum_{v=-\infty}^{\infty} \sqrt{\frac{2 E_{b}}{N_{c} T_{s}} \sum_{k=1}^{N_{u}} \sum_{n=1}^{N_{c}} h_{k, n} b_{k}(v) c_{k, n} u_{T_{s}}\left(t-v T_{s}-\xi_{k}\right)} \\
\times \cos \left(w_{n} t+\phi_{k, n}\right),
\end{gathered}
$$

where $\phi_{k, n}=\theta_{k, n}+\varphi_{k, n}-w_{n} \xi_{k}, \xi_{k}$ is the time misalignment of user $k$ with respect to user 1 (the reference user), $h_{k, n}$ and $\varphi_{k, n}$ are the frequency-domain subcarrier fading gain and phase for the $n$th subcarrier, respectively, and $\eta(t)$ is the additive white Gaussian noise (AWGN). The decision variable $U$ of user 1 is

$$
\begin{aligned}
U & =\int_{0}^{T_{s}} r(t) \sum_{n=1}^{N_{c}} c_{1, n} \cos \left(w_{n} t+\phi_{1, n}\right) \alpha_{1, n} d t \\
& =D+I+J+\eta,
\end{aligned}
$$

where $D$ and $\eta$ are the desired signal and noise components, respectively, $I$ and $J$ are the MUI components, and $\alpha_{1, n}$ is the combiner coefficient for the $n$th subcarrier of user 1 . Without loss of generality, we abbreviate $h_{1, n}$ as $h_{n}$ and $\alpha_{1, n}$ as $\alpha_{n}$. For MRC, the combiner coefficient $\alpha_{n}=h_{n}$. Then the desired signal component $D=\sqrt{E_{b} T_{s} / 2 N_{c}} \sum_{n=1}^{N_{c}} h_{n}^{2}$, and the noise component $\eta$ has zero mean and variance $\left(N_{0} T_{s} / 4\right) \sum_{n=1}^{N_{c}} h_{n}^{2}$. For simplicity in analysis, the uplink MUI is divided into two parts: $I$ is the MUI from the same subcarrier of other users while $J$ is the MUI from other subcarriers of other users [3], that is,

$$
\begin{aligned}
I= & \sqrt{\frac{E_{b} T_{s}}{2 N_{c}}} \sum_{k=2}^{N_{u}} \sum_{n=1}^{N_{c}} h_{k, n} \alpha_{n} \cos \left(\phi_{k, n}-\phi_{1, n}\right) \\
& \times\left[b_{k}(-1) c_{k, n} c_{1, n} \xi_{k}+b_{k}(0) c_{1, n} c_{k, n}\left(T_{s}-\xi_{k}\right)\right], \\
J= & \sqrt{\frac{E_{b} T_{s}}{2 N_{c}} \sum_{k=2}^{N_{u}} \sum_{n=1}^{N_{c}} \sum_{q=1, q \neq n}^{N_{c}} h_{k, n} \alpha_{n}} \\
& \times\left\{\int_{0}^{\xi_{k}} b_{k}(-1) c_{k, n} c_{1, n} \cos \left(\left(w_{n}-w_{q}\right) t+\phi_{k, n}-\phi_{1, n}\right) d t\right. \\
& \left.\quad+\int_{\xi_{k}}^{T_{s}} b_{k}(0) c_{k, n} c_{1, n} \cos \left(\left(w_{n}-w_{q}\right) t+\phi_{k, n}-\phi_{1, n}\right) d t\right\},
\end{aligned}
$$

where $b_{k}(0)$ and $b_{k}(-1)$ represent the current and previous data bits of the $k$ th user, respectively. Since the user data and fading parameters of different users are uncorrelated, the summands in (4) are uncorrelated too. Even though the subcarrier fading gain $h_{k, n}$ of the same user may be correlated to some extent, the summands in (4) in this case are still uncorrelated due to the presence of other uncorrelated variables such as phase $\phi_{k, n}$ in the equations. Moreover, since the number of summands in (4) are very large (e.g., $N_{c}$ can be at least 64 and $N_{u}$ can be as large as $N_{c}$ ), both $I$ and $J$ are the summations of large number of uncorrelated terms. Hence, central limit theorem (CLT) can be applied to approximate $I$ and $J$ as Gaussian random variables (RVs) [13]. It is shown in [3] that $I$ and $J$ have zero mean and variance given by

$$
\begin{gathered}
\operatorname{var}(I)=\frac{E_{b} T_{s}\left(N_{u}-1\right) \sigma^{2}}{3 N_{c}} \sum_{n=1}^{N_{c}} h_{n}^{2}, \\
\operatorname{var}(J)=\frac{E_{b} T_{s}\left(N_{u}-1\right) \sigma^{2}}{4 N_{c} \pi^{2}} \sum_{n=1}^{N_{c}} h_{n}^{2} \sum_{i=1, i \neq n}^{N_{c}}(i-n)^{-2},
\end{gathered}
$$

where $\sigma^{2}$ is the subcarrier fading power of other users. Thus the BER of an MC-CDMA uplink channel conditioned on 
the set of subcarrier fading amplitudes $\left\{h_{n}\right\}$ is

$$
\begin{aligned}
& P_{e} \mid\left\{h_{n}\right\} \\
& =Q\left(\frac{\sum_{n=1}^{N_{c}} h_{n}^{2}}{\sqrt{\left(\frac{2\left(N_{u}-1\right) \sigma^{2}}{3}+\frac{N_{c}}{2 E_{b} / N_{0}}\right) \sum_{n=1}^{N_{c}} h_{n}^{2}+\frac{\left(N_{u}-1\right) \sigma^{2}}{2 \pi^{2}} \sum_{n=1}^{N_{c}} \sum_{i=1, i \neq n}^{N_{c}}(i-n)^{-2} h_{n}^{2}}}\right)
\end{aligned}
$$

where $Q(\cdot)$ is Gaussian $Q$-function. The average BER $P_{e}$ can then be obtained by averaging (7) over the joint distribution function (jdf) of $\left\{h_{n}\right\}$, that is,

$$
P_{e}=\int_{0}^{\infty} \cdots \int_{0}^{\infty}\left(P_{e} \mid\left\{h_{n}\right\}\right) \mathrm{jdf}\left(\left\{h_{n}\right\}\right) d h_{1} \cdots d h_{N_{c}} .
$$

The multidimensional integration in (8) is not easy to evaluate even by using Monte Carlo integration. This is because the number of subcarriers is usually large (e.g., 64 in IEEE 802.11a wireless LAN systems) and the subcarrier fading gains are usually correlated.

\subsection{Simplification of $B E R$ formula}

The presence of $\sum_{i=1, i \neq n}^{N_{c}}(i-n)^{-2}$ in (6) results in different dependence on $h_{n}$ in the variance expressions of $I, J$, and $\eta$, thus complicating the analysis of the uplink BER. However, we noticed that its value does not vary much for different values of $n$, hence it can be approximated as a constant $a$ whose value only depends on $N_{c}$, that is,

$$
\sum_{i=1, i \neq n}^{N_{c}} \frac{1}{(i-n)^{2}} \simeq \frac{1}{N_{c}} \sum_{n=1}^{N_{c}} \sum_{i=1, i \neq n}^{N_{c}} \frac{1}{(i-n)^{2}}=a .
$$

Later we will show using simulation results that the effect of the approximation made in (9) on the BER is negligible. With this approximation, the term $\sum_{n=1}^{N_{c}} h_{n}^{2}$ becomes a common factor in the $\operatorname{var}(I)$ expression (5), the $\operatorname{var}(J)$ expression (6), and the variance expression of $\eta$. Thus the conditional uplink BER expression in (7) can be simplified to

$$
P_{e} \mid\left\{h_{n}\right\}=Q\left(\sqrt{\frac{\sum_{n=1}^{N_{c}} h_{n}^{2}}{\frac{2\left(N_{u}-1\right) \sigma^{2}}{3}+\frac{\left(N_{u}-1\right) \sigma^{2} a}{2 \pi^{2}}+\frac{N_{c}}{2 E_{b} / N_{0}}}}\right) .
$$

Denoting

$$
\beta=\sum_{n=1}^{N_{c}} h_{n}^{2}
$$

the BER can be obtained by averaging (10) over the probability density function (pdf) of the combined subcarrier fading variable $\beta$, that is,

$$
P_{e}=\int_{0}^{\infty} Q(\sqrt{\nu \beta}) f(\beta) d \beta,
$$

where $f(\cdot)$ denotes the pdf and $\nu$ is given by

$$
v=\left[\frac{2\left(N_{u}-1\right) \sigma^{2}}{3}+\frac{\left(N_{u}-1\right) \sigma^{2} a}{2 \pi^{2}}+\frac{N_{c}}{2} \frac{N_{0}}{E_{b}}\right]^{-1} .
$$

Comparing (12) and (8), the dimension of integration is reduced from $N_{c}$ to one, provided that the pdf of $\beta$ can be obtained. However, in general, it is not easy to find the pdf of $\beta$ for larger number of subcarriers whose fading gains may be correlated, and/or different subcarriers may have different fading characteristics. We will circumvent this problem by transforming the subcarrier-domain integration in (12) into a path-domain integration. This will be elaborated in the next section.

\subsection{Time- and frequency-domain equivalence of MRC Output}

The CIR of a multipath fading channel with $N_{p}$ resolvable paths is typically represented using the tapped delay line model as

$$
g(t)=\sum_{l=1}^{N_{p}} g_{l} \exp \left(j \psi_{l}\right) \delta\left(t-\tau_{l}\right),
$$

where $g_{l}, \psi_{l}$, and $\tau_{l}$ are the fading envelope, phase, and delay of the lth path, respectively.

Denoting the complex subcarrier fading gains as a vector $\tilde{\mathbf{h}}$ with length $N_{c}$, and the complex path fading gains as a vector $\tilde{\mathbf{g}}$ with length $N_{p}$, then $\tilde{\mathbf{h}}$ is related to $\tilde{\mathbf{g}}$ by discrete Fourier transform (DFT) $[14,15]$, that is,

$$
\tilde{\mathbf{h}}=\mathbf{W} \tilde{\mathbf{g}} \text {, }
$$

where

W

$$
=\left(\begin{array}{cccc}
1 & 1 & \cdots & 1 \\
e^{-j 2 \pi \tau_{1} / T_{s}} & e^{-j 2 \pi \tau_{2} / T_{s}} & \cdots & e^{-j 2 \pi \tau_{N_{p}} / T_{s}} \\
e^{-j 4 \pi \tau_{1} / T_{s}} & e^{-j 4 \pi \tau_{2} / T_{s}} & \cdots & e^{-j 4 \pi \tau_{N_{p}} / T_{s}} \\
\vdots & \vdots & \vdots & \vdots \\
e^{-j\left(N_{c}-1\right) 2 \pi \tau_{1} / T_{s}} & e^{-j\left(N_{c}-1\right) 2 \pi \tau_{2} / T_{s}} & \cdots & e^{-j\left(N_{c}-1\right) 2 \pi \tau_{N_{p}} / T_{s}}
\end{array}\right) .
$$

The term $\sum_{n=1}^{N_{c}} h_{n}^{2}$ in (10) can now be represented as

$$
\sum_{n=1}^{N_{c}} h_{n}^{2}=\tilde{\mathbf{h}}^{H} \tilde{\mathbf{h}}=\tilde{\mathbf{g}}^{H} \mathbf{W}^{H} \mathbf{W} \tilde{\mathbf{g}}=N_{c} \tilde{\mathbf{g}}^{H} \tilde{\mathbf{g}}=N_{c} \sum_{l=1}^{N_{p}} g_{l}^{2}
$$

due to the fact that $\mathbf{W}^{H} \mathbf{W}=N_{c} \mathbf{I}_{N_{p}}$, where $\mathbf{I}_{N_{p}}$ denotes an $N_{p} \times N_{p}$ identity matrix and the superscript $H$ denotes the matrix Hermitian transpose operator. Expression (17) signifies that MRC of subcarrier fading is equivalent to MRC of 
path fading; hence the BER expression in (12) can now be rewritten as

$$
P_{e}=\int_{0}^{\infty} Q\left(\sqrt{\nu N_{c} \gamma}\right) f(\gamma) d \gamma
$$

where

$$
\gamma=\sum_{l=1}^{N_{p}} g_{l}^{2}
$$

Compared to (12), (18) is much easier to compute because the pdf of $\gamma$ is generally easier to analyze than the pdf of $\beta$ for the following reasons:

(i) most practical channels are characterized and represented in the form of CIR or power delay profiles $[16,17]$, which are more directly applicable to (18) than (12);

(ii) the number of significant channel paths $N_{p}$ is normally much less than the number of subcarriers $N_{c}$. For example, $N_{c}$ can be 64 for the IEEE 802.11a wireless LAN standard, or as large as 2056 for the European digital video broadcasting (DVB) standard, while $N_{p}$ in practical wireless communication systems is normally less than $10[16,17]$;

(iii) last but not least, fading among the subcarriers is normally correlated (even for channels with independent fading paths $[2,10])$. This increases the complexity in determining the $\mathrm{pdf}$ of $\beta$.

Therefore, we will use (18) and the pdf of the combined path fading variable $\gamma$ to formulate $P_{e}$ in the next section.

\section{BER FORMULATIONS FOR DIFFERENT FADING CHANNELS}

Notice that (18) is equal to the BER expression for a conventional time-domain MRC system, so the problem now is to find the pdf of $\gamma$, which is the MRC output of the multiple fading paths. To be most general, we will consider the pdf of $\gamma$ with arbitrarily correlated paths in Rayleigh, Rician, and Nakagami fading channels. Rayleigh fading is discussed as a special case of Nakagami or Rician fading.

\subsection{Nakagami fading channels with independent paths}

In this subsection, we model the fading path gains $\left\{g_{l}\right\}$ as independent Nakagami-distributed RVs. Nakagami fading distribution, also known as $m$-distribution, is widely adopted for modelling fading channels because of its good fit to empirical measurements $[18,19,20]$, as well as the tractability it renders to BER evaluation [21]. A variety of fading effects can be modelled as Nakagami fading with different $m$ parameters, including Rayleigh fading as a special case when $m$ equals 1 . The Nakagami- $m$ distribution is given by

$$
f\left(g_{l}\right)=\frac{2}{\Gamma\left(m_{l}\right)}\left(\frac{m_{l}}{\Omega_{l}}\right)^{m_{l}} g_{l}^{2 m_{l}-1} \exp \left[-\left(\frac{m_{l}}{\Omega_{l}}\right) g_{l}^{2}\right],
$$

where $\Omega_{l}=E\left[g_{l}^{2}\right]$ and $m_{l}=E^{2}\left[g_{l}^{2}\right] / E\left[\left(g_{l}^{2}-E\left[g_{l}^{2}\right]\right)^{2}\right] . \Gamma(\cdot)$ is the Euler gamma function and $E[\cdot]$ denotes statistical expectation. Since the square of Nakagami RV $\gamma_{l}=g_{l}^{2}$ follows the gamma distribution

$$
f\left(\gamma_{l}\right)=\frac{\left(m_{l} / \Omega_{l}\right)^{m_{l}} e^{-\left(m_{l} / \Omega_{l}\right) \gamma_{l}} \gamma_{l}^{m_{l}-1}}{\Gamma\left(m_{l}\right)}
$$

the MRC signal $\gamma=\gamma_{1}+\gamma_{2}+\cdots+\gamma_{N_{p}}$ can be viewed as the summation of $N_{p}$ number of gamma variables.

If $\left\{\gamma_{l}\right\}$ are independent with identical $m_{l} / \Omega_{l}$ for all values of $l, \gamma$ follows exactly another gamma distribution with new values of $m$ and $\Omega$ given by [5]

$$
\begin{aligned}
& m=\sum_{l=1}^{N_{p}} m_{l}, \\
& \Omega=\sum_{l=1}^{N_{p}} \Omega_{l} .
\end{aligned}
$$

For independent $\left\{\gamma_{l}\right\}$ with nonidentical $m_{l} / \Omega_{l}$, the exact pdf of $\gamma$ becomes much more complicated to derive. In [22], we have shown that the combined output $\gamma$ in this case can be adequately approximated as a new gamma-distributed RV. For this resultant gamma distribution, its power $\Omega$ is given by (23), while its $m$ parameter value can be derived through moment matching to be

$$
m=\frac{\left(\sum_{l=1}^{N_{p}} \Omega_{l}\right)^{2}}{\sum_{l=1}^{N_{p}}\left(\Omega_{l}^{2} / m_{l}\right)} .
$$

For equal $m_{l} / \Omega_{l},(24)$ is reduced to (22).

Substituting (21) into the BER formula (18) with appropriate $m$ and $\Omega$ parameters, we have

$$
\begin{aligned}
P_{e} & =\int_{0}^{\infty} Q\left(\sqrt{\nu N_{c} \gamma}\right) \frac{(m / \Omega)^{m} e^{-(m / \Omega) \gamma} \gamma^{m-1}}{\Gamma(m)} d \gamma \\
& =\frac{\left(\frac{m}{\Omega} \frac{1}{v}\right)^{m} \Gamma\left(\frac{1}{2}+m\right)_{2} F_{1}\left(m, \frac{1}{2}+m ; 1+m,-\frac{m}{\Omega} \frac{1}{v}\right)}{2 \sqrt{\pi} \Gamma(1+m)},
\end{aligned}
$$

where ${ }_{2} F_{1}(\cdot)$ is the hypergeometric function and $v$ is given in (13).

\subsection{Nakagami fading channels with correlated paths}

Although statistical independence among the diversity branches is desired in MRC systems, there are cases where this assumption is not valid $[23,24]$. Hence we consider Nakagami fading channels with correlated paths in this subsection. Dual-branch MRC system with correlated Nakagami fading branches is discussed in $[5,24]$. The study is further generalized to arbitrary number of diversity branches in [23], subject to the conditions of identical branch parameters, that is, $m_{l}$ and $\Omega_{l}$ are the same for all values of $l$. Also, the results of [23] are only applicable to constant or exponential branch correlation models. In [25], arbitrary branch correlation is 
studied, but the analysis is limited to identical and integervalued $m_{l}$ parameters across the branches. In $[21,26]$, noninteger $m_{l}$ values are considered, but the $m_{l}$ parameters must still be identical across the branches.

In [27], we propose an approach to obtain the fading statistics of correlated $\left\{\gamma_{l}\right\}$ without any constraints on the fading parameters and correlations of the channel paths. In this approach, Cholesky decomposition is used to transform correlated gamma RVs into linear combinations of independent gamma RVs. Specifically, denote $\boldsymbol{\gamma}=\left[\gamma_{1}, \ldots, \gamma_{N_{p}}\right]^{T}$ as the set of correlated gamma variables with covariance matrix $\mathbf{C}_{\gamma}=E\left[\gamma \gamma^{T}\right]-E[\boldsymbol{\gamma}] E\left[\boldsymbol{\gamma}^{T}\right]$. By Cholesky decomposition, $\mathbf{C}_{y}=\mathbf{L} \mathbf{L}^{T}$, where $\mathbf{L}$ is a lower triangular matrix with $(j, i)$ th element denoted as $l_{j i}$. Let $\mathbf{w}=\left[\begin{array}{llll}w_{1} & w_{2} & \cdots & w_{N_{p}}\end{array}\right]^{T}$ be a set of independent gamma RVs with identity covariance matrix $\mathrm{C}_{\mathrm{w}}$.

Next, let

$$
\gamma=\mathbf{L w}
$$

or equivalently

$$
\begin{aligned}
& \gamma_{1}=l_{11} w_{1}, \\
& \gamma_{2}=l_{21} w_{1}+l_{22} w_{2}, \\
& \vdots \\
& \gamma_{l}=\sum_{i=1}^{l} l_{l i} w_{i}, \\
& \vdots \\
& \gamma_{N_{p}}=\sum_{i=1}^{N_{p}} l_{N_{p} i} w_{i} ;
\end{aligned}
$$

then the covariance matrix of $\mathbf{L w}$ is

$$
E\left[\mathbf{L}(\mathbf{w}-E[\mathbf{w}])(\mathbf{w}-E[\mathbf{w}])^{T} \mathbf{L}^{T}\right]=\mathbf{L L}^{T}=\mathbf{C}_{\boldsymbol{\gamma}} .
$$

Therefore, the correlated path variables $\gamma$ have been transformed to weighted sums of independent variables $\mathbf{w}$ with weights given by (26) or (27), without affecting the path correlations.

By matching the moments of both sides of (27) progressively from top down, the $m$-parameter $m_{w, i}$ and the power $\Omega_{w, i}$ of the elements $w_{i}$ in $\mathbf{w}$ can be obtained iteratively using the following equations:

$$
\begin{gathered}
m_{w, 1}=m_{1}, \\
m_{w, i}=l_{i i}^{-2}\left(\sum_{q=1}^{i-1} l_{i q} \sqrt{m_{w, q}}-\Omega_{i}\right)^{2}, \\
\Omega_{w, i}=\sqrt{m_{w, i} .}
\end{gathered}
$$

Summing up both sides of (27) then gives the resultant combined output

$$
\gamma=\sum_{l=1}^{N_{p}}\left(w_{l} \sum_{i=1}^{l} l_{l i}\right)
$$

Since $\left\{w_{i}\right\}$ are independent, it follows from our earlier analysis in this paper that $\gamma$ can be approximated as a new gamma distributed RV with $\Omega$ given by (23) and $m$ given by

$$
m=\Omega^{2}\left[\sum_{l=1}^{N_{p}} m_{w, l}^{-1}\left(\Omega_{w, l} \sum_{i=1}^{l} l_{l i}\right)^{2}\right]^{-1} .
$$

It can be shown that the $m$-parameter expression given in (31) reduces to (24) and (22) for independent paths with nonidentical $m_{l} / \Omega_{l}$ and identical $m_{l} / \Omega_{l}$ ratios, respectively. Hence (31) is a more general expression.

Finally, it should be clear from the preceding analyses that a closed-form BER formula for MC-CDMA uplink channel with MRC in Nakagami fading channel without any constraint on the fading parameters and correlations of the channel paths is realized in (25), with $v, \Omega$, and $m$ given by (13), (23), and (31), respectively. Furthermore, with $m_{l}=1$ for the fading paths, (25) becomes applicable to channels with Rayleigh fading paths.

\subsection{Rician fading channels with independent or correlated paths}

The Rician distribution is another popular fading model for signal envelops received in channels with direct line-of-sight (LOS) or specular component [28]. When the LOS component is absent, the Rician fading distribution will be reduced to Rayleigh. The BER of the MRC diversity system with Rayleigh diversity branches are available in $[28,29]$. However, for Rician fading and especially correlated Rician fading branches, the results in $[28,30,31]$ are complicated as they may include hypergeometric functions or sum of integrals of Bessel functions. In this section, we will derive a new one-dimensional integral BER expression based on the characteristic function (CF) [32] of the combined output.

The Rician fading path gains $g_{l}$ follow the pdf expression

$$
\begin{aligned}
f\left(g_{l}\right)= & \frac{2\left(K_{l}+1\right) g_{l}}{\Omega_{l}} \exp \left(-K_{l}-\frac{\left(K_{l}+1\right) g_{l}{ }^{2}}{\Omega_{l}}\right) \\
& \times I_{0}\left(2 \sqrt{\frac{K_{l}\left(K_{l}+1\right)}{\Omega_{l}}} g_{l}\right),
\end{aligned}
$$

where $\Omega_{l}=E\left[g_{l}^{2}\right], I_{0}(\cdot)$ is the zeroth-order modified Bessel function of the first kind, and $K_{l}$ is the Rician $K$ factor [28]. When $K_{l}=0$, (32) reduces to Rayleigh fading distribution.

It is well known that for Rician fading channel, the complex channel gain can be represented as complex Gaussian RVs, that is,

$$
\tilde{\mathbf{g}}=\left[g_{1} \exp \left(j \psi_{1}\right), \ldots, g_{N_{p}} \exp \left(j \psi_{N_{p}}\right)\right]^{T}=\mathbf{X}_{c}+j \mathbf{X}_{s} .
$$

Define $\mathbf{X}=\left[\mathbf{X}_{c} ; \mathbf{X}_{s}\right]$, where $\mathbf{X}_{c}$ and $\mathbf{X}_{s}$ are $N_{p} \times 1$ real Gaussian random vectors, $\boldsymbol{\mu}=E[\mathbf{X}]$ as the mean vector, and $\mathbf{C}_{x}$ as the covariance matrix of $\mathbf{X}$. The CF of $\gamma=\sum_{l=1}^{N_{p}} g_{l}^{2}$ is given in [32] as follows:

$$
\Psi_{\gamma}(j \omega)=\frac{\exp \left[j \omega \sum_{k=1}^{2 N_{p}}\left(\epsilon_{k}^{2} /\left(1-2 j \omega \lambda_{k}\right)\right)\right]}{\prod_{k=1}^{2 N_{p}}\left(1-2 j \omega \lambda_{k}\right)^{1 / 2}},
$$




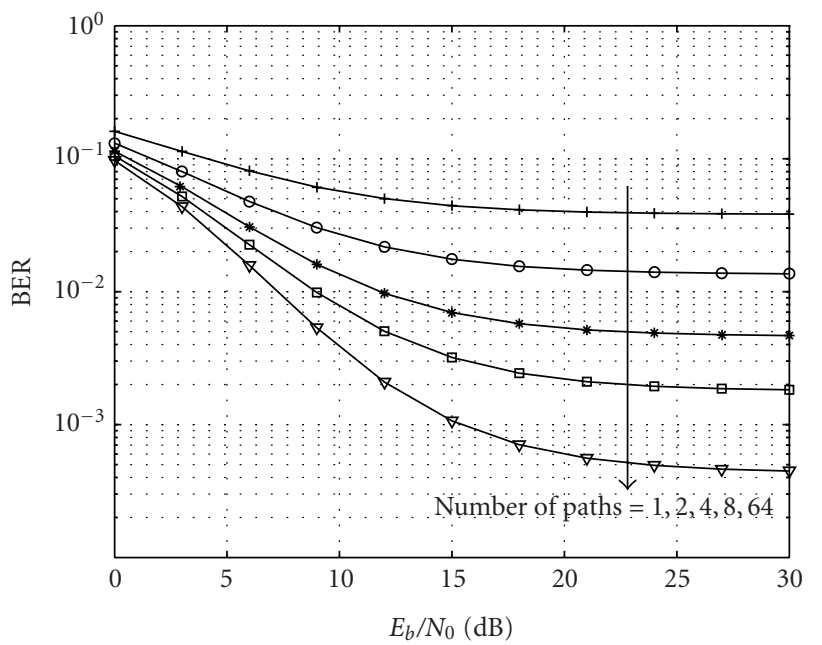

Solid lines: our results

Markers: results from [10]

FIGURE 1: Comparison of BER values computed using (25) in this paper and BER values taken from [10, Figure 4] (64 subcarriers, 12 users, i.i.d. Rayleigh paths).

where $\lambda_{k}$ are the eigenvalues of $\mathbf{C}_{x}, \mathbf{C}_{x}=\mathbf{V} \boldsymbol{\Lambda} \mathbf{V}^{T}, \boldsymbol{\Lambda}=$ $\operatorname{diag}\left(\lambda_{1}, \lambda_{2}, \ldots, \lambda_{2 N_{p}}\right)$, and $\epsilon_{k}$ is given by $\left[\epsilon_{1}, \epsilon_{2}, \ldots, \epsilon_{2 N_{p}}\right]^{T}=$ $\mathbf{V}^{T} \boldsymbol{\mu}$.

By utilizing an alternative expression for the Gaussian Qfunction given in [33], that is,

$$
Q(x)=\frac{1}{\pi} \int_{0}^{\pi / 2} \exp \left(-\frac{x^{2}}{2 \sin ^{2} \phi}\right) d \phi
$$

and with the CF of $\gamma$ given in (34), the BER expression (18) of MC-CDMA uplink with MRC in correlated Rician fading channel can now be obtained by the new equation as shown:

$$
P_{e}=\frac{1}{\pi} \int_{0}^{\pi / 2} \Psi_{\gamma}\left(-\frac{\nu N_{c}}{\sin ^{2} \phi}\right) d \phi
$$

Since only one-dimensional integration is involved and the integration limits are finite, (36) can be easily evaluated numerically. Also, (36) is general enough to cover the case of independent paths with $\mathbf{C}_{x}$ being a diagonal matrix, and the Rayleigh fading case with $\boldsymbol{\mu}=\mathbf{0}$.

\section{RESULTS AND DISCUSSIONS}

To demonstrate the validity and simplicity of our proposed BER formulation approaches, we compare our results with that in [10], which considers channels with i.i.d. Rayleigh fading paths. Laplace transform and residual method are used in [10] to compute the pdf of $\sum_{n=1}^{N_{c}} h_{n}^{2}$ and the resultant BER expression is in one-dimensional integration form. In contrast, our BER expression for this case is the closed-form formula in (25). In Figure 1, the analytical BER values com-

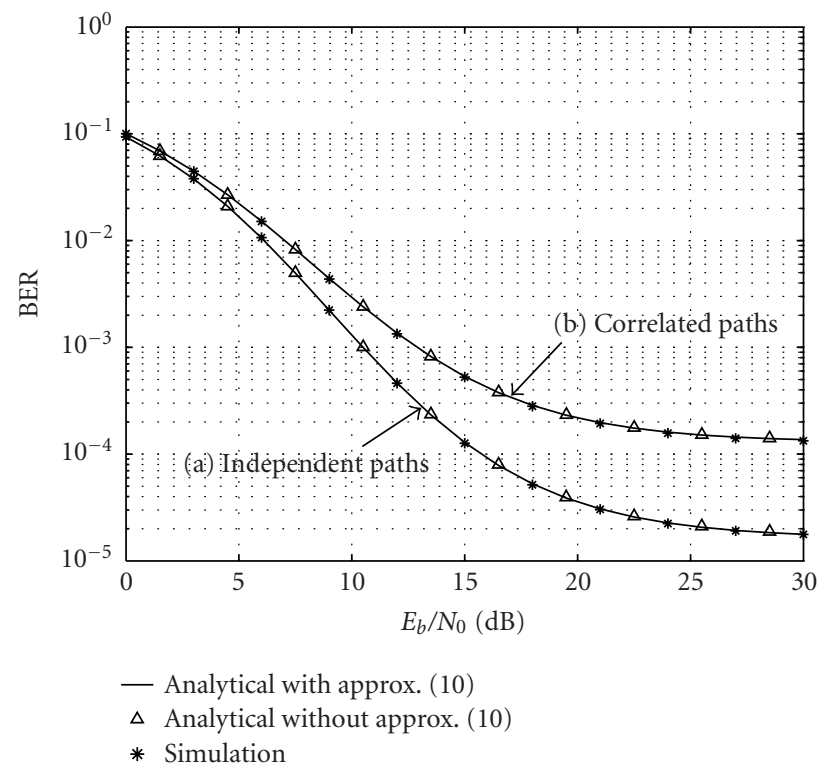

FIgure 2: Performance of MC-CDMA uplink with MRC (128 subcarriers, 10 users) in a channel with 3 correlated Rician fading paths, $K=\left[\begin{array}{lll}5 & 3 & 2\end{array}\right], \Omega=\left[\begin{array}{lll}0.4 & 0.35 & 0.25\end{array}\right]$. Channel (a) contains independent paths with $\mathbf{C}_{x}=\mathbf{I}_{6}$; channel (b) contains correlated paths with

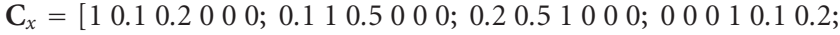
$0000.110 .5 ; 0000.20 .51]$.

puted using (25) in this paper are compared with the BER values taken from [10, Figure 4]. Both sets of BER values are found to match exactly.

In Figures 2 and 3, we use computer simulations of asynchronous MC-CDMA uplink with 128 subcarriers and 10 active users to verify our BER formulas for different fading conditions. There are three types of BER plots in Figures 2 and 3: one simulation plot and two analytical plots (obtained with or without the approximation made in (9)). Figure 2 is for a channel with independent or correlated Rician fading paths with randomly selected $K_{l}, \Omega_{l}$, and correlation values. Figure 3 is for Nakagami channels consisting of independent paths with identical $m_{l} / \Omega_{l}$ ratio, or correlated paths with unequal $m_{l} / \Omega_{l}$ ratios. Detailed channel specifications are given in the respective figure captions.

The "analytical with approximation (9)" plots in Figures 2 and 3 are obtained by using (36) and (25), respectively, while the "analytical without approximation (9)" plots are obtained by Monte Carlo integration of the conditional BER given in (7). Both Figures 2 and 3 show that the analytical BER values with or without approximation (9) are indistinguishable, hence the effect of the approximation made in (9) on the system BER values is negligible. Although not shown in this paper, the same verification has also been carried out for other values of subcarrier number $N_{c}$ and fading parameters, for example, $N_{c}$ from 16 to 256 , Rician $K$ factor from 0 to 20, and Nakagami $m$ parameter from 1 to 20. The same conclusion that the effect of the approximation made in (9) on the system BER is insignificant can be reached. 


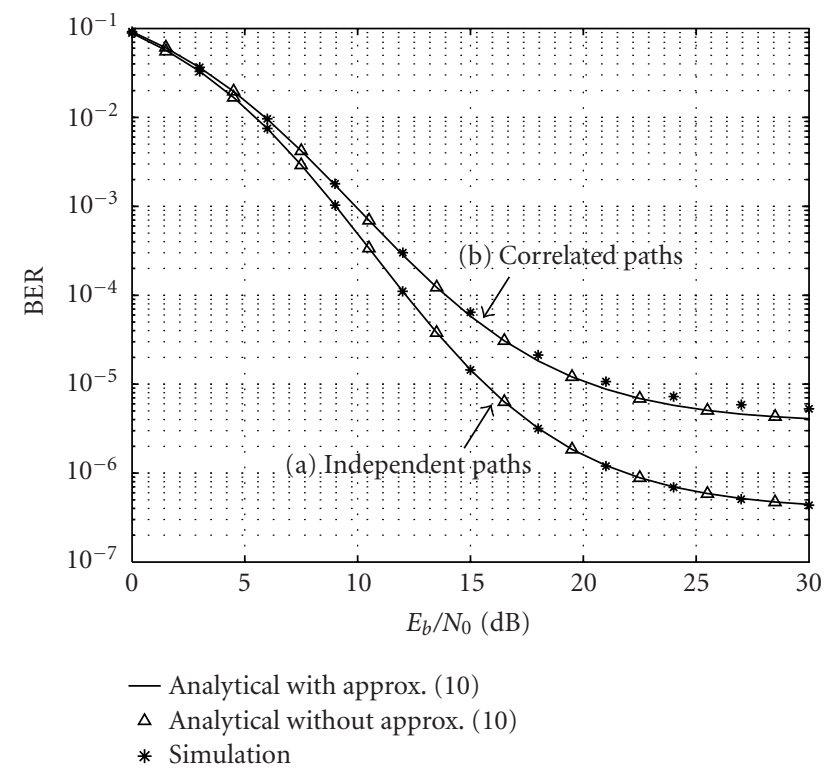

FIgURe 3: Performance of MC-CDMA uplink with MRC (128 subcarriers, 10 users) in a channel with (a) 4 independent Nakagami fading paths, $m=$ [9 554 2 $]$, $\Omega=\left[\begin{array}{llll}0.45 & 0.25 & 0.2 & 0.1\end{array}\right]$; (b) 3 correlated Nakagami fading paths, $m=\left[\begin{array}{lll}9 & 7 & 3\end{array}\right], \Omega=\left[\begin{array}{lll}0.5 & 0.3 & 0.2\end{array}\right], \mathbf{C}_{\gamma}=\left[\begin{array}{ll}1 & 0.4\end{array}\right.$ $0.3 ; 0.410 .7 ; 0.30 .71$ ].

For Nakagami fading channels, if the fadings in different channel paths are independent and have identical $m_{l} / \Omega_{l}$ ratio, the MRC output $\gamma$ follows an exact gamma distribution. Hence, as seen for the channel condition (a) in Figure 3, both the simulation and analytical BER plots match very well. On the other hand, when the fadings in different channel paths have different $m_{l} / \Omega_{l}$ ratios or are correlated, $\gamma$ is only approximately gamma-distributed. Hence, some mismatch can be observed between the analytical and simulated BER plots for the channel condition (b) in Figure 3. The approximation error associated with the pdf of $\gamma$ has been discussed in detail in $[22,27]$. As concluded in these papers, for most cases, the approximation error is very small and acceptable.

As seen in Figures 1, 2, and 3, the MC-CDMA uplink exhibits the familiar error floor at high $E_{b} / N_{0}$ level due to the MUI power predominating over the AWGN power. With our simple uplink BER expressions of (36) and (25), the error floor can be easily evaluated by setting $E_{b} / N_{0}$ in (13) to infinity. Figure 4 shows the dependence of the error floor values on the number of users in channels with correlated Rician and Nakagami paths. The Rician channel model has the same parameters as the channel condition (b) in Figure 2, while the Nakagami channel model is the same as the channel condition (b) in Figure 3. The Rician channel suffers higher error floor because its channel paths are subject to more severe fading than the Nakagami channel.

Furthermore, with our closed-form or one-dimensional integral BER formulas, the effect of various system or channel parameters on the system performance can also be ob-

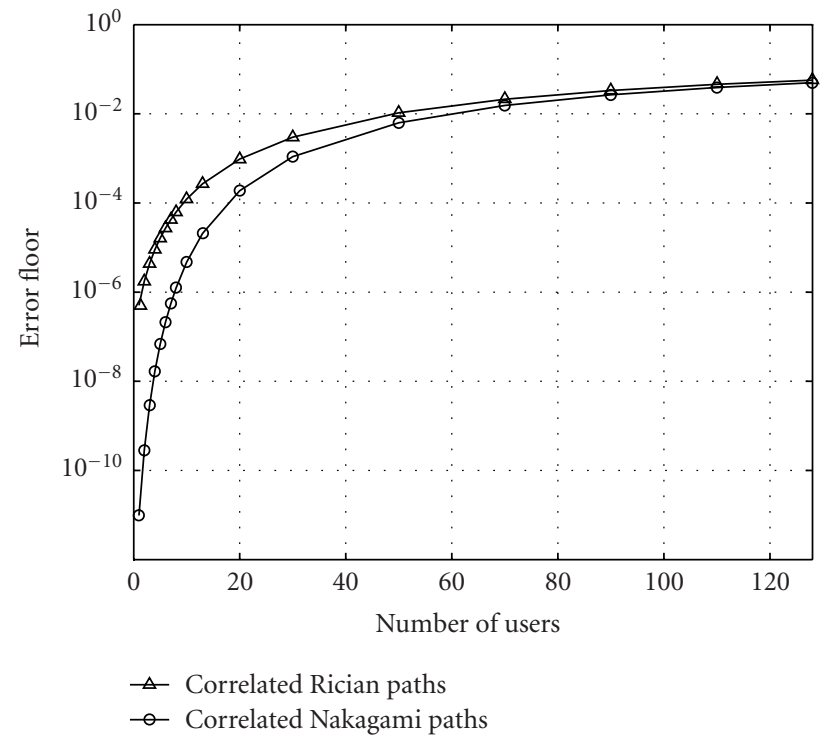

FIGURE 4: Error floor values versus number of users for MC-CDMA uplink with MRC. Number of subcarriers = 128 .

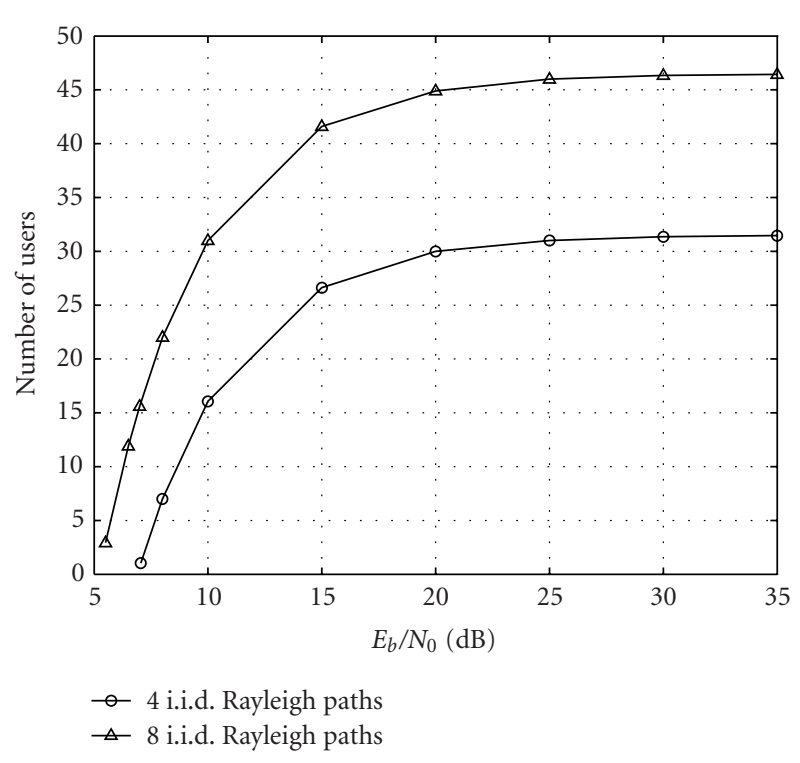

FIGURE 5: User capacity of MC-CDMA uplink with MRC in channels with i.i.d. Rayleigh fading paths (target $\mathrm{BER}=10^{-3}, 128$ subcarriers).

tained with ease. As an example, Figure 5 shows how many users the MC-CDMA system can support in order to meet a target BER of $10^{-3}$ in channels with 4 or 8 i.i.d. Rayleigh paths. Such user capacity results can be easily obtained from (25) by simple numerical root finding. Besides, our earlier analysis predicts that the larger the number of channel paths, the more diversity the MC-CDMA system can achieve. This explains why the channel with more paths in Figure 5 can accommodate more users. 


\section{CONCLUSIONS}

In this paper, we present a way to obtain the analytical BER of MC-CDMA uplink with MRC in channels with correlated Rayleigh, Rician, or Nakagami fading paths. We first achieved a simplified signal model for the MRC output and established its time-frequency equivalence, which states that combining the subcarriers using MRC has exactly the same effect as combining the channel paths using MRC. This principle is exploited to achieve very simplified BER formulas based on (CIR) information of the MC-CDMA system under study. A new closed-form BER formula is derived for channels with correlated Nakagami fading paths using the techniques of Cholesky decomposition and gamma pdf approximation. The BER formula is exact if all the channel paths have identical $m_{l} / \Omega_{l}$ ratio; otherwise, it is approximate, but nonetheless adequately accurate. For channels with correlated Rician fading paths, the associated analytical BER formula is derived by appropriate function mapping based on $\mathrm{CF}$ and an alternative form of the Gaussian $Q$-function. The resultant BER formula contains only one-dimensional integration and hence can be easily integrated numerically.

\section{REFERENCES}

[1] A. C. McCormick and E. A. Al-Susa, "Multicarrier CDMA for future generation mobile communication," Electronics \& Communication Engineering Journal, vol. 14, no. 2, pp. 52-60, 2002.

[2] S. Hara and R. Prasad, "Overview of multicarrier CDMA," IEEE Communications Magazine, vol. 35, no. 12, pp. 126-133, 1997.

[3] X. Gui and T. S. Ng, "Performance of asynchronous orthogonal multicarrier CDMA system in frequency selective fading channel," IEEE Transactions on Communications, vol. 47, no. 7, pp. 1084-1091, 1999.

[4] W. C. Jakes Jr., Ed., Microwave Mobile Communications, Wiley, New York, NY, USA, 1974.

[5] E. K. Al-Hussaini and A. M. Al-Bassiouni, "Performance of MRC diversity systems for the detection of signals with Nakagami fading," IEEE Transactions on Communications, vol. 33, no. 12, pp. 1315-1319, 1985.

[6] N. Yee, J. P. Linnartz, and G. Fettweis, "Multi-carrier CDMA in indoor wireless radio networks," in Proc. IEEE International Symposium on Personal, Indoor and Mobile Radio Communications (PIMRC'93), pp. 109-113, Yokohama, Japan, September 1993.

[7] S. Kaiser, "On the performance of different detection techniques for OFDM-CDMA in fading channels," in Proc. IEEE Global Telecommunications Conference (GLOBECOM '95), vol. 3, pp. 2059-2063, Singapore, November 1995.

[8] R. L. Gouable and M. Helard, "Performance of single and multi-user detection techniques for a MC-CDMA system over channel model used for HIPERLAN2," in Proc. IEEE International Symposium on Spread Spectrum Techniques and Applications (ISSSTA '00), vol. 2, pp. 718-722, Parsippany, NJ, USA, September 2000.

[9] Q. Shi and M. Latva-aho, "An exact error floor for downlink MC-CDMA in correlated Rayleigh fading channels," IEEE Communications Letters, vol. 6, no. 5, pp. 196-198, 2002.

[10] J. Park, J. Kim, S. Choi, N. Cho, and D. Hong, "Performance of MC-CDMA systems in non-independent Rayleigh fading," in Proc. IEEE International Conference on Communications (ICC '99), pp. 506-510, Vancouver, BC, Canada, June 1999.
[11] Z. Li and M. Latva-aho, "Simple analysis of MRC receivers for MC-CDMA systems in fading channels," in Proc. International Conferences on Info-Tech and Info-Net (ICII '01), vol. 2, pp. 560-565, Beijing, China, October 2001.

[12] Q. Shi and M. Latva-aho, "Exact bit error rate calculations for synchronous MC-CDMA over a Rayleigh fading channel," IEEE Communications Letters, vol. 6, no. 7, pp. 276-278, 2002.

[13] A. Papoulis and S. U. Pillai, Probability, Random Variables and Stochastic Processes, McGraw-Hill, New York, NY, USA, 4th edition, 2002.

[14] O. Edfors, M. Sandell, J.-J. van de Beek, S. K. Wilson, and P. O. Borjesson, "OFDM channel estimation by singular value decomposition," IEEE Transactions on Communications, vol. 46, no. 7, pp. 931-939, 1998.

[15] B. Yang, K. B. Letaief, R. S. Cheng, and Z. Cao, "Channel estimation for OFDM transmission in multipath fading channels based on parametric channel modeling," IEEE Transactions on Communications, vol. 49, no. 3, pp. 467-479, 2001.

[16] T. Ojanpera and R. Prasad, Wideband CDMA for Third Generation Mobile Communications, Artech House Publishers, Boston, Mass, USA, 2001.

[17] M. Patzold, Mobile Fading Channels, Wiley, New York, NY, USA, 2002.

[18] G. L. Turin, W. S. Jewell, and T. L. Johnston, "Simulation of urban vehicle-monitoring systems," IEEE Transactions on Vehicular Technology, vol. 21, no. 1, pp. 9-16, 1972.

[19] H. Suzuki, "A statistical model for urban radio propagation," IEEE Transactions on Communications, vol. 25, no. 7, pp. 673680, 1977.

[20] A. Wojnar, "Rayleigh, Rice and Nakagami-in search of efficient models of fading radio channels," in Int. Wroclaw Symp. Electromagnetic Compatibility, pp. 797-802, Wroclaw, Poland, 1988.

[21] Q. T. Zhang, "Maximal-ratio combining over Nakagami fading channels with an arbitrary branch covariance matrix," IEEE Transactions Vehicular Technology, vol. 48, no. 4, pp. 1141-1150, 1999.

[22] K. Zhang, Z. Song, and Y. L. Guan, "A generalized model for the combined output statistics of MRC diversity systems in Nakagami fading channels," in Int. Symp. Communication Systems, Networks and Digital Signal Processing (CSNDSP '02), Staffordshire University, UK, July 2002.

[23] V. A. Aalo, "Performance of maximal-ratio diversity systems in a correlated Nakagami-fading environment," IEEE Transactions on Communications, vol. 43, no. 8, pp. 2360-2369, 1995.

[24] G. Fedele, L. Izzo, and M. Tanda, "Dual diversity reception of M-ary DPSK signals over Nakagami fading channels," in IEEE 6th Int. Symp. Personal, Indoor and Mobile Radio Communication (PIMRC '95), pp. 1195-1201, Toronto, Canada, September 1995.

[25] P. Lombardo, G. Fedele, and M. M. Rao, "MRC performance for binary signals in Nakagami fading with general branch correlation," IEEE Transactions on Communications, vol. 47, no. 1, pp. 44-52, 1999.

[26] M.-S. Alouini, A. Abdi, and M. Kaveh, "Sum of gamma variates and performance of wireless communication systems over Nakagami-fading channels," IEEE Transactions on Vehicular Technology, vol. 50, no. 6, pp. 1471-1480, 2001.

[27] K. Zhang, Z. Song, and Y. L. Guan, "Cholesky decomposition model for correlated MRC diversity systems in Nakagami fading channels," in IEEE 56th Vehicular Technology Conference (VTC'02 Fall), vol. 3, pp. 1515-1519, Vancouver, BC, Canada, September 2002.

[28] M. K. Simon and M.-S. Alouini, Digital Communication over Fading Channels: A Unified Approach to Performance Analysis, Wiley, New York, NY, USA, 2000. 
[29] J. G. Proakis, Digital Communications, McGraw-Hill, New York, NY, USA, 4th edition, 2001.

[30] D. D. N. Bevan, V. T. Ermolayev, and A. G. Flaksman, "Coherent multichannel reception of binary modulated signals with dependent Rician fading," IEE Proceedings Communications, vol. 148, no. 2, pp. 105-111, 2001.

[31] W. C. Lindsey, "Error probabilities for Rician fading multichannel reception of binary and N-ary signals," IEEE Transactions on Information Theory, vol. 10, no. 4, pp. 339-350, 1964.

[32] R. K. Mallik and M. Z. Win, "Error probability of binary NFSK and DPSK with postdetection combining over correlated Rician channels," IEEE Transactions on Communications, vol. 48, no. 12, pp. 1975-1978, 2000.

[33] M.-S. Alouini and A. J. Goldsmith, "A unified approach for calculating error rates of linearly modulated signals over generalized fading channels," IEEE Transactions on Communications, vol. 47, no. 9, pp. 1324-1334, 1999.

Keli Zhang received the B.Eng. degree in electrical and electronic engineering from Nanyang Technological University, Singapore. Since 2001, she has been working towards the Ph.D. degree in wireless communications at the School of Electrical and Electronic Engineering (EEE) in Nanyang Technological University, Singapore. Her research interests include channel modeling for MCM systems, performance analysis of MC-CDMA, diversity combining.

Yong Liang Guan received his B.Eng. and Ph.D. degrees from the National University of Singapore and Imperial College of Science, Technology and Medicine, University of London, respectively. He is currently an Assistant Professor in the School of Electrical and Electronic Engineering (EEE), Nanyang Technological University (NTU), Singapore. He is also the Program Director for the Wireless Network Research Group

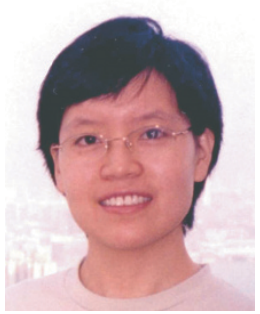
in the Positioning and Wireless Technology Center (PWTC), and the Deputy Director of the Center for Information Security, NTU. His research interests include multicarrier modulation, Turbo and space-time coding/decoding, channel modelling, and digital multimedia watermarking.

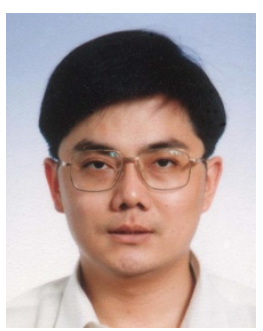

